\title{
El impacto de la Covid-19 en el trabajo de bibliotecas y archivos: nuevas formas de trabajo y de comunicación con los usuarios
}

\author{
Debate coordinado por BLANCA SAN JOSÉ MONTANO, ELENA LÓPEZ DE LA FUENTE y ANA \\ NASEIRO RAMUDO \\ Vocalía de Publicaciones de SEDIC
}

\begin{abstract}
El debate analiza a través de una serie de preguntas planteadas a diversos profesionales de archivos y bibliotecas españolas, sus inquietudes, situación y los cambios experimentados en la relación con sus usuarios durante la pandemia de la Covid-19.
\end{abstract}

Pandemia, Archivos, Bibliotecas, Covid-19, Comunicación, Usuarios, Servicios, Canales comunicación, Digitalización, Formatos electrónicos, Espacios físicos.

\section{Introducción}

Desde que el día 14 de marzo el Gobierno decretó el Estado de Alarma con la finalidad de hacer frente a la expansión de la pandemia mundial provocada por el Covid-19, las Bibliotecas y Archivos han cambiado sus formas de trabajo para adecuarlos a las nuevas necesidades y formas de vida.

Como decía Darwin sobreviven los que se adaptan mejor al cambio y nuestra profesión ha sido y es siempre, un ejemplo de habituación a las exigencias del contexto y muy especialmente a los requerimientos de sus usuarios.

En muchos casos, nuestro rápido paso al teletrabajo ha sido facilitado por una experiencia previa marcada por un largo camino ya andado hacia la transformación digital con la previa adaptación de nuestros soportes mediante la digitalización y el empleo de servicios en línea con los usuarios.

Con el fin de obtener una amplia visión acerca de la situación actual generada por la pandemia en nuestras formas de trabajo, las diferentes necesidades de los usuarios y las adaptaciones de las Instituciones, hemos seleccionado a 6 profesionales de diferentes Instituciones y especializaciones (Biblioteca de ciencias de la salud, Archivo estatal, Biblioteca Universitaria, Biblioteca escolar, Biblioteca pública y Biblioteca especializada en economía), con el fin de que opinen sobre su actual situación laboral después del confinamiento y la desescalada, para que expongan su visión, ideas, opiniones e interés respecto a la actual realidad profesional.

Bajo el principio de que el acceso abierto a los resultados de investigación acelera el avance del conocimiento, todos los contenidos de la edición electrónica de CLIP se distribuyen bajo una licencia de uso y distribución Creative Commons Reconocimiento-NoComercialCompartirlgual 3.0 España (CC BY-NC-SA 3.0 ES). 


\section{Cuestionario}

\section{1. ¿Cómo ha respondido su Institución al Estado de alarma? ¿Estaba preparada su biblioteca/archivo para realizar teletrabajo o tuvo que adaptarse a la nueva situación adoptando herramientas para poder realizarlo?}

Montaña Vivas Jiménez (Biblioteca especializada en ciencias de la salud)

La gerencia del Área de Salud de Cáceres se vio sorprendida, como la mayoría de las instituciones, por la repentina decisión de la promulgación del estado de alarma en España.

La medida condujo a desarrollar inmediatamente normas de funcionamiento de uso interno y carácter personal (suspensión de vacaciones y permisos, organización del teletrabajo para trabajadores no esenciales y vulnerables, etc.) y otras de carácter sanitario como las "Normas de actuación de los responsables de unidades y servicios en casos de contactos laborales COVID-19", el "Protocolo de prevención y protección tras las medidas de contención por la pandemia de Coronavirus COVID-19" o los sucesivos "Planes de contingencia del Área de Salud de Cáceres", entre otras, que llevaron a desbordar la administración, que estaba ya mermada por los trabajadores infectados y de baja. Finalmente una parte, nuestro caso particular, no tuvimos esta opción y hemos trabajado normalmente durante y después del estado de alarma.

A pesar de ello creo que nuestra biblioteca dispone de programas que hubieran hecho posible, aunque fuera en parte, el teletrabajo (préstamo interbibliotecario, recursos de Saludteca y de la Web de la Biblioteca, Repositorio institucional, etc.), y también herramientas de acceso remoto o conexión $\mathrm{VNC}$ con el equipo.

Un problema que tenemos y hubiéramos tenido en el caso del teletrabajo es que nuestra Intranet no permite el intercambio de archivos a través de las nubes comerciales, ni el acceso a este tipo de herramientas necesarias para ello, debido a los cortafuegos de seguridad implementados. Otros inconvenientes son el tipo de equipo y software y las conexiones que tenemos en nuestras casas, para desarrollar el trabajo eficazmente.

José Luis Muñoz Romano (Archivo estatal)

Como la mayoría de archivos nos vimos obligados a cerrar las instalaciones del Archivo, no obstante, seguimos dando servicio telemático a los usuarios externos que así lo requerían a través del buzón de correo y el formulario de consulta de nuestra página web. Nos mantuvimos así hasta el 9 de junio (fase 2 de la desescalada).

En nuestro caso diría que estábamos semi-preparados, ya que el Ministerio contaba con un programa de teletrabajo aprobado antes de que empezara la pandemia. Este hecho ha facilitado la instalación de los programas pertinentes por parte de unos informáticos que ya se manejaban en este terreno. En el caso del personal, sí hemos tenido ciertas dificultades al no recibir ningún tipo de formación.

Fernando Martín Rodríguez (Biblioteca universitaria)

En cierta medida todas las actividades profesionales, sociales, etc. han tratado de responder lo mejor que han podido a esta situación tan excepcional. En el caso de las Bibliotecas Universitarias ha facilitado enormemente el alto grado de digitalización en varias áreas. Por una parte en lo referido a contenidos. Del presupuesto de fondo bibliográfico, según los datos estadísticos de REBIUN correspondientes al año 2019 las Bibliotecas Universitarias y Científicas dedicaron más de 122 
millones de euros en la suscripción y compra de fondo bibliográfico, y de estos el 85\% se dedicó a colecciones electrónicas.

En lo que se refiere a desarrollo tecnológico, durante años los sistemas de acceso off-campus a los recursos digitales han permitido seguir prestando servicio a investigadores y alumnos. También ha sido posible realizar teletrabajo gracias a los sistemas de automatización de las bibliotecas, que permiten conectarse en remoto a través de aplicaciones web y a los sistemas de acceso remoto a las estaciones de trabajo. Incluso muchas Bibliotecas disponían de sistemas de telefonía móvil, por lo que la comunicación telefónica ha podido continuar. Quizá ha sido necesario durante este tiempo adquirir equipos informáticos portátiles para garantizar este teletrabajo.

\section{María José Lamas (Biblioteca escolar)}

Como coordinadora de la biblioteca escolar de un colegio de Educación Primaria, opino que el estado de alarma nos supuso una alteración imprevista en la dinámica diaria de funcionamiento para la que el centro escolar, en todas sus dimensiones, no estaba preparado. Así, fue preciso modificar improvisadamente muchos de sus aspectos para seguir realizando el servicio habitual de la biblioteca con el fin de seguir cumpliendo sus objetivos en la mayor medida posible.

\section{Carlos García-Romeral Pérez (Biblioteca pública)}

Los Servicios de Lectura Pública están integrados por Bibliotecas Públicas, Bibliometro, Telebiblioteca, Bibliobuses y servicios de extensión bibliotecaria. El 80\% de las tareas que realizan los más de 400 empleados que conforman su plantilla, son de proximidad y de relación directa al ciudadano (usuario), y muy pocas, se pueden realizar de forma telemática. A pesar de ello se estudiaron qué tareas se podrían realizar y cómo podrían realizarse. Lo más importante al principio fue organizar los Recursos Humanos y Tecnológicos en el "Estado de Alarma" del 13 de marzo de 2020. La adaptación a esta nueva situación fue sobre la marcha: ordenadores, conexiones, mantenimiento y creación de grupos etc... Es complicado gestionar un grupo tan numeroso de empleados en un momento tan difícil y que a su vez se sintieran que estaban realizando un trabajo relacionado con el día a día de su trabajo habitual. Se asignaron y coordinaron tareas manteniendo la misma organización jerárquica, potenciando las ya existentes relaciones horizontales con grupos de trabajo habituales: selección, guías de lectura, efemérides. etc. Todo ello impulsando los sistemas de comunicación telemáticos.

Si tenemos que enumerar rasgos que definan la profesión de bibliotecario es el de la "capacidad de adaptación a los cambios" y el de "reinventar las tareas para adaptarlas a una nueva realidad" teniendo como objetivo, el mantener la relación con los usuarios de los respectivos servicios y satisfacer sus necesidades informativas y de lectura. Sentirnos integrados en la sociedad y que el usuario sienta que estamos con él.

\section{José Antonio Sánchez Montero (Biblioteca especializada en economía)}

Desde un primer momento, a comienzos de marzo de 2020, todo el personal de la Comisión Nacional de los Mercados y la Competencia (CNMC) fue enviado a casa a continuar su actividad por medios telemáticos. Evidentemente, la biblioteca y centro de documentación no estaba preparada para un cambio de escenario tan abrupto en la prestación de sus servicios, sobre todo en todo lo referente a la colección impresa; no obstante, la decidida apuesta digital en las políticas de gestión de información desarrolladas con anterioridad a ese momento por la Biblioteca y Centro de Documentación CNMC ("Plan integral de gestión de información y del conocimiento", materializado en la Biblioteca Digital $\mathrm{CNMC}$ ), ayudaron bastante a amortiguar el impacto del cambio. Al principio, la principal dificultad 
fue la escasez de licencias VPN y la adaptación hardware/software de los equipos informáticos personales en los hogares, situaciones que se solventaron en las siguientes semanas. Lógicamente, también fue necesario un periodo de adaptación en el sistema y flujos de trabajo, así como en los medios de comunicación interna del equipo de trabajo de la biblioteca.

\section{2. ¿Ha estado realizando su trabajo habitual de forma virtual o ha estado desempeñando otro tipo de trabajo diferente?}

Montaña Vivas Jiménez (Biblioteca especializada en ciencias de la salud)

En mi caso el trabajo que he desarrollado ha sido el habitual, en la biblioteca, sin que en ningún momento nos destinasen a otras tareas. Sin embargo la Biblioteca, como servicio, se ofreció a la dirección para ayudar en lo que estimasen oportuno. En un momento determinado desde la fotocopiadora-escáner se digitalizaron documentos de pedidos para el servicio de almacén, que se había tenido que cerrar temporalmente. Pero siempre de forma voluntaria.

José Luis Muñoz Romano (Archivo estatal)

Hasta el 1 de junio mi trabajo fue 100\% telemático, posteriormente hemos ido alternando una semana en presencial y otra en no presencial, así hasta el 29 de junio. Al finalizar ese periodo, cambié por tres días en presencial y dos en no presencial al conciliar.

Fernando Martín Rodríguez (Biblioteca universitaria)

En ese proceso de adaptación urgente que todos hemos vivido, hemos tratado de ser lo más flexibles posible y atender las necesidades que ha sufrido la propia Universidad. Por ejemplo, y en mi caso concreto, hemos dado respuesta a una necesidad relativa a la lectura de tesis durante el estado de alarma de forma remota y hemos trabajado conjuntamente con la Escuela de Doctorado adaptando para ello herramientas de la Biblioteca en tiempo record o incluso prestando equipos portátiles a los alumnos desde las casas particulares del personal de la biblioteca.

En cuanto a las formas de trabajar, creo que de forma generalizada podemos decir que el Teams y el Zoom se han incorporado a nuestras vidas sin darnos cuenta y ya resultan imprescindibles...

María José Lamas (Biblioteca escolar)

Durante el confinamiento y posterior período de educación telemática en el tercer trimestre del pasado curso escolar, las funciones de la biblioteca se limitaron a la realización de préstamos a través de las tutorías de las distintas aulas, pues, al no haber clases presenciales, no funcionaba como espacio de encuentro para la realización de las actividades habituales: charlas, debates, conferencias, talleres de teatro, poesía, actividades grupales, y un largo etcétera. No obstante, se publicitaron las nuevas adquisiciones de material bibliográfico y consejos sobre bibliografía adecuada a los diferentes niveles educativos y sobre temáticas concretas susceptibles de ser utilizada por alumnado y profesorado.

Carlos García-Romeral Pérez (Biblioteca pública) 
El trabajo habitual de los empleados que conforman los Servicios Públicos de Lectura de la Comunidad de Madrid, se centra en el ciudadano (usuarios). Se mantuvieron aquellas tareas que podían realizarse de forma telemática: depuración del catálogo bibliográfico, depuración de las bases de datos de lectores, selección de materiales para el incremento de las bibliotecas, mantenimiento del contacto con los proveedores (libros y audiovisuales), mantenimiento de los email institucionales de las bibliotecas, contestación de llamadas telefónicas (se desviaron los teléfonos fijos a móviles), etc. Todo ello en función de las diferentes categorías profesionales.

Las bibliotecas públicas en muchos casos se han transformado en apoyo emocional a los usuarios sobre todo el servicio de Telebiblioteca.

José Antonio Sánchez Montero (Biblioteca especializada en economía)

En general, tanto mi trabajo como el del resto del equipo de biblioteca, cambió muy poco con el teletrabajo, salvo la lógica excepción de la imposibilidad de realizar tareas relacionadas con la gestión de la colección en formato papel. En consecuencia, sí se produjo un mayor énfasis en todos los aspectos relacionados con la gestión de recursos electrónicos de información y la formación de usuarios en estos.

\section{3. ¿Qué tipos de servicios han estado prestando a sus usuarios y cómo? ¿Son servicios ya existentes o de nueva creación??}

Montaña Vivas Jiménez (Biblioteca especializada en ciencias de la salud)

- Lectura en sala. A los escasos usuarios presenciales -profesionales- que hubo durante el estado de alarma les hemos prestado el servicio de lectura en sala: con medidas de higiénicas, limitación de aforo, redistribución del mobiliario y equipos según normas de distanciamiento, etc.

- Búsquedas bibliográficas, las habituales aunque por interés de estudio de la biblioteca separamos las de temática COVID del resto.

- Préstamo Interbibliotecario: el habitual, aunque por interés de estudio de la biblioteca separamos las de temática COVID del resto.

- Repositorio científico.

- Servicios de la Biblioteca Virtual.

- Servicio de información a través del Blog y redes sociales de Saludteca. en sala.

El servicio de Biblioteca de Pacientes se cerró y quedaron suspendidos el préstamo y la lectura

Como servicio de "nueva creación" podríamos mencionar el repositorio colaborativo "COVID-19 por especialidades y temas" (https://sites.google.com/view/covid19-porespecialidades/página-principal?authuser $=0$ ) que pusimos en marcha en marzo del 2020 y en el que se subieron y clasificaron cientos de artículos y documentos de toda índole a medida que los usuarios los demandaban y los bibliotecarios participantes los localizábamos. El repositorio se creó en un site de Google y nosotros, a su vez, lo pusimos tanto en la web de la biblioteca del Área de Salud de Cáceres, como en la de la biblioteca virtual Saludteca, a disposición de nuestros usuarios.

José Luis Muñoz Romano (Archivo estatal) 
Durante el confinamiento no se prestó documentación a las unidades productoras ni tampoco se admitieron las consultas en sala. El servicio fundamental fue atender a los usuarios externos (investigadores y ciudadanos) que demandaban información sobre nuestros fondos (Obras públicas y Vivienda).

Fernando Martín Rodríguez (Biblioteca universitaria)

En el caso concreto de la Biblioteca de la Universidad de Burgos, hemos reforzado la compra de bibliografía recomendada en formato electrónico, especialmente la dirigida a los alumnos y durante la apertura progresiva de la Biblioteca hemos habilitado un servicio de escaneado y envío de documentos con las garantías legales en lo referido a la propiedad intelectual. También hemos mejorado el sistema de gestión de la bibliografía recomendada y ampliada la oferta de cursos de formación on-line para los usuarios.

\section{Carlos García-Romeral Pérez (Biblioteca pública)}

Se han seguido prestando los servicios que habitualmente se ofertan en las bibliotecas, pero cambiando el formato y sobre todo desarrollando y potenciando el uso de la plataforma ebiblioMadrid y la colección digital madrileña. También se realizaron altas de lectores para que pudieran utilizar ebiblio. El correo electrónico de los diferentes servicios se transformó en una pieza clave de relación con los usuarios, que tenían/en medios y habilidades para poder conectarse. La pandemia COVID-19 ha agudizado más la BRECHA social, cultural y digital

Los Servicios de Lectura Pública tienen la misión de socializar y democratizar el acceso a la información y a la cultura. Los servicios Telemáticos son claves para aquellos usuarios que tienen los medios económicos (pago de conexión a internet, dispositivos -ordenadores, Tablet...) además de las habilidades informáticas (sabe conectarse y donde hacerlo).

Entre las diferentes tareas que se han realizado por los empleados de las bibliotecas ha estado la de mantener viva la información al ciudadano, a través del Portal del Lector (http://www.madrid.org/cs/Satellite?pagename=PortalLector\%2FPage\%2FPLEC home). Se creó el site "Año Galdós”, para participar desde los servicios públicos de lectura en las acciones culturales que se celebran bajo el lema "Galdós vive Galdós (1920-2020)" y manteniendo el espacio de información sobre la APP en site "Tableteando", donde se informa de las diferentes aplicaciones enfocadas a la educación y la cultura; gestionado por un grupo de trabajo horizontal.

Se ha mantenido el espacio "La Biblioteca Recomienda" selección de materiales de lectura y que se pueden encontrar en la plataforma ebiblioMadrid; así como el "Espacio Joven".

Se potenciaron los Clubes de Lectura Virtuales que ya se celebraban de forma habitual con acceso e inscripción desde el Portal del Lector, manteniendo de esta forma vivo el espíritu de los clubes de lectura tradicionales (presenciales) de los Servicio de Lectura Pública.

Además se han mantenido las actividades de fomento de la lectura, cambiando el canal de difusión. Para los más pequeños, con la iniciativa El cuento de cada tarde, desde el 23 de marzo hasta el 30 de abril, se emitió en las redes sociales una narración diaria de 20 minutos, se reproducía de esta forma los Cuentacuentos. En el mes de mayo se desarrolló la campaña ACTívateEnCasa, en la que ponía a disposición de los niños y niñas diariamente un video de contenido cultural e información sobre literatura infantil.

Se han celebrado las efemérides más importantes del "Estado de Alarma": 23 de abril Día del Libro; el 2 de abril Día del Libro Infantil y Juvenil en el que se homenajeó a Gianni Rodari y el 21 de marzo Día de la Poesía. 
Siguiendo la estela de un libro de Gianni Rodari, se están organizando por los trabajadores en relación con los usuarios Cuentos por teléfono.

Se han potenciado los espacios telemáticos de difusión de la cultura, la ciencia y el fomento de la lectura; pero no son posibles sin espacios de proximidad. Los Servicio de Lectura Pública son ahora más que nunca los garantes de la democratización de la cultura y punto de apoyo emocional de los ciudadanos, es el principal espacio para paliar la soledad y desindividualizar la sociedad.

José Antonio Sánchez Montero (Biblioteca especializada en economía)

Los únicos servicios que no se pudieron prestar durante el confinamiento fueron el préstamo de obras impresas de la Biblioteca CNMC y el préstamo interbibliotecario, suspendido en esos momentos. El resto son servicios que ya se prestaban anteriormente con normalidad gracias a la existencia de la Biblioteca Digital CNMC, un repositorio interno sobradamente conocido por los usuarios ya que cuenta con 15 años de antigüedad en la institución, y que aúna el acceso de todos los recursos electrónicos de CNMC: libros, informes, estadísticas, revistas electrónicas, portales especializados, bases de datos, etc. Entre los servicios que siguieron prestándose con normalidad: edición y envío de boletines informativos de biblioteca, difusión selectiva de información, servicio de alertas e-mail, servicio de referencia e información bibliográfica, gestión de adquisiciones y suscripciones digitales, acceso a prensa digital, etc. El único servicio puesto en marcha durante la pandemia ha sido la contratación de una plataforma de préstamo de e-books, que ofreciera una solución digital a la imposibilidad de acceso a muchos de los libros en formato impreso existentes en la Biblioteca. Como novedad durante la fase de confinamiento, y sólo en algunos casos especiales debido a la falta de recursos, se puso en marcha un sistema de préstamo y entrega a domicilio de documentos en formato papel de la Biblioteca.

\section{4. ¿Qué canales de comunicación han utilizado con los usuarios?}

Montaña Vivas Jiménez (Biblioteca especializada en ciencias de la salud)

En nuestro caso hemos utilizado los habituales: correo electrónico y formularios de contacto de la Biblioteca del Área de Salud de Cáceres y de la Biblioteca Virtual Saludteca.

Sin embargo lo que hemos priorizado ha sido nuestra comunicación a través del Blog "Píldoras" https://mariamontanavivas.wordpress.com y de la cuenta de Twitter@Saludteca, que han tenido una actividad frenética en este periodo y que han servido para dar a conocer y compartir la ingente cantidad de documentos y publicaciones que se sucedían a diario procedentes del ámbito nacional e internacional.

\section{José Luis Muñoz Romano (Archivo estatal)}

Fundamentalmente el buzón de correo y el formulario de consulta de la página web del Archivo. Para anunciar los protocolos de reapertura optamos por publicarlo en nuestra página web y publicitarlo a través del perfil institucional del Ministerio en Twitter. Sugerimos también el canal de noticias de Telegram pero no se nos tuvo en cuenta.

Fernando Martín Rodríguez (Biblioteca universitaria) 
Hemos mantenido los canales habituales de comunicación, excepto el presencial. Gracias a la telefonía móvil los bibliotecarios han seguido prestando atención telefónica. Sí que se ha tratado de utilizar con mayor intensidad las redes sociales a través de los perfiles abiertos por la biblioteca y hemos notado un incremento de las consultas que los usuarios han realizado a través del servicio de atención por Whatsapp.

\section{Carlos García-Romeral Pérez (Biblioteca pública)}

Portal del Lector, página oficial de la Subdirección General del Libro, en la que no sólo se difunden los servicios, actividades... sino que informa a los ciudadanos de los diferentes cambios en estos servicios, Redes Sociales, ECorreos (Correos electrónicos) de los diferentes servicios, teléfonos de las bibliotecas, envío masivos de información a los usuarios a través de listas de distribución, además de la cartelería visible en las puertas de las bibliotecas.

José Antonio Sánchez Montero (Biblioteca especializada en economía)

Además del portal interno de la Biblioteca en la intranet CNMC que cuenta con medios particulares como RSS y formularios de contacto y solicitudes, los principales canales de comunicación han sido el correo electrónico por un lado, y por otro el chat, la llamada telefónica y la videoconferencia que de forma integrada proporciona Teams, una herramienta de comunicación que ya se usaba en CNMC previamente a la pandemia. También se ha visto incrementado el uso de otros canales para compartir ficheros como Google Drive o We'Transfer.

\section{5. ¿Cómo han realizado o van a realizar la desescalada? ¿Qué normativas han seguido? ¿Han elaborado normas/plan propio o la institución?}

\section{Montaña Vivas Jiménez (Biblioteca especializada en ciencias de la salud)}

Hemos ido estudiando las normas y recomendaciones, tanto nacionales como internacionales, que afectaban al uso y funcionamiento de las bibliotecas en tiempo de pandemia y adaptándolas a nuestro entorno hospitalario, para elaborar unas normas propias y adaptadas al Plan de Contingencia del Área de Salud de Cáceres.

Particularmente construimos y publicamos la infografía "Cómo realizar la devolución y el uso de documentos en las bibliotecas de hospital durante la COVID-19".

En el momento actual seguimos manteniendo las mismas normas relativas a la higiene, distanciamiento, ventilación, uso de los ordenadores, etc. de la biblioteca. Sin embargo, hemos reducido el tiempo de la cuarentena de los documentos y todavía no hemos reabierto la biblioteca de pacientes.

\section{José Luis Muñoz Romano (Archivo estatal)}

Sí, elaboramos un primer protocolo de reapertura del archivo que contempla la legislación que regulaba el Estado de Alama y los servicios a prestar por los Archivos -recordemos que nos consideraban servicio esencial desde la misma Fase 0-; el tipo de atención que íbamos a prestar atención preferentemente telemática, consulta presencial con cita previa, distancia interpersonal de 2 metros, uso obligatorio de mascarilla, guantes, lavado de manos, señalización dentro de la sala para mantener la distancia interpersonal, etcétera-; el establecimiento de una cuarentena para el material 
consultado; incluso cómo recoger la documentación en las distintas naves dependientes de nuestro Servicio fijando como protocolo que solo acuda un trabajador y un conductor, guardando las medidas de seguridad, higiene y distancia social en el vehículo. Finalmente añadimos un cronograma de la desescalada, indicando el número de personas y el tipo de servicio (telemático / presencial) establecido para cada fase.

Fernando Martín Rodríguez (Biblioteca universitaria)

Inicialmente todos hemos estado pendientes de lo que indicaban las diferentes normas del Ministerio de Sanidad y de las Comunidades Autónomas. También han sido útiles las recomendaciones elaboradas por REBIUN y por diferentes asociaciones profesionales. Conforme se ha ido avanzando en la desescalada, se ha ido aplicando lo establecido en los planes por cada Universidad, y en esto sí que hemos encontrado poca homogeneidad. Cada Biblioteca ha ido al ritmo marcado por los comités COVID de cada Institución.

Hay que tener en cuenta que el objetivo principal en la educación superior es el de preservar y mantener la enseñanza de forma presencial y en esto la Biblioteca ha adoptado medidas más restrictivas en los servicios prestados, de tal forma que se han reducido los aforos a las instalaciones tratando de cumplir la distancia social, se ha restringido el acceso a las bibliotecas exclusivamente a los miembros de la Comunidad Universitaria y se han cancelado algunos servicios como las salas de trabajo en grupo, el uso de ordenadores y la programación de carácter cultural (exposiciones, actividades de teatro o música, por ejemplo).

Carlos García-Romeral Pérez (Biblioteca pública)

Se siguen los protocolos de la Consejería de Sanidad de la Comunidad de Madrid, para cada una de las fases y situaciones. Se han elaborado protocolos de funcionamiento interno de los servicios tanto bibliotecarios como los servicios generales (limpieza y seguridad) adaptados a los diferentes Servicios Públicos de Lectura.

No sólo se han elaborado protocolos de funcionamiento interno sino que se han ido adaptando las instalaciones (aires acondicionados) a la situación de pandemia en que nos encontramos por el COVID-19. Se ha dotado además a todos los trabajadores de los EPIs (Equipos de Protección Individual) necesarios para realizar sus tareas y a los usuarios de la información sanitaria para que puedan acceder sin riesgo a las bibliotecas. Desde el 15 de junio con aforos restringidos y controlados se reactiva el libre acceso a salas de lectura y colecciones de las bibliotecas.

José Antonio Sánchez Montero (Biblioteca especializada en economía)

En un principio, se había previsto el retorno escalonado al trabajo presencial durante el mes de septiembre, mediante turnos y con una afluencia máxima de un 33\% del personal en cada unidad. Sin embargo, la evolución de la pandemia desde finales de agosto y septiembre, ha impedido finalmente la vuelta presencial al trabajo. Durante estos meses de pandemia, la Biblioteca ha compaginado en todo momento el cumplimiento de la normativa interna de CNMC relativa a medidas de seguridad, sanitarias, teletrabajo, etc. con las Recomendaciones para la reapertura al público de las bibliotecas que iba implementando de forma sucesiva el Ministerio de Cultura y Deportes en cada una de las fases de la desescalada (Subdirección General de Coordinación Bibliotecaria. Dirección General del Libro y Fomento de la Lectura). En este sentido, desde junio se decidió la asistencia presencial un día a la semana para ir resolviendo los temas pendientes de la colección física y volver a la normalidad total del servicio de biblioteca. En relación a la elaboración de un plan propio, nuestra Subdirección elaboró unas Recomendaciones internas basadas en la 
normativa interna de CNMC y la del Ministerio de Cultura y Deportes, que finalmente no han sido difundidas por la evolución negativa de la pandemia tras el verano, con el nuevo aumento de contagios y consiguientes restricciones a la movilidad, optando finalmente por una comunicación bilateral con los usuarios para su aplicación.

\section{En su opinión ¿qué consecuencias en cuanto a servicios de atención a usuarios, espacios,} formas de trabajo, etc...van a permanecer después de la vuelta a la "Nueva Normalidad"?

\section{Montaña Vivas Jiménez (Biblioteca de ciencias de la salud)}

Probablemente lo que más se verá afectado son los espacios físicos de las bibliotecas mientras tengamos que seguir cumpliendo las normas de higiene y seguridad impuestas y convenientes.

Respecto al resto del trabajo, las bibliotecas llevan ya muchos años ofreciendo y prestando servicios remotos a través de la web o de las bibliotecas virtuales de las comunidades.

Después de esta experiencia creo que las bibliotecas que no tienen servicios de información y difusión en redes sociales deberían plantearse seriamente ponerlos en marcha, porque como en este caso, cuando las bibliotecas se cierran tienen que tener activos otros canales de comunicación y presencia en el espacio virtual que, si no ocupamos nosotros, pueden ocupar otros.

\section{José Luis Muñoz Romano (Archivo estatal)}

Para los usuarios externos la cita previa, al agilizar y mejorar nuestro servicio ya que nos permite programar mejor nuestros recursos, evitando incómodas esperas que antes sí sucedían cuando los usuarios se presentaban directamente sin avisar en la sala de atención al público. Hay que tener en cuenta que el servicio de automoción tarda 48 horas en dar servicio y buena parte de la documentación no se encuentra depositada en la Sede Central.

Para los usuarios internos sin duda la digitalización completa de los expedientes personales que solicitan en préstamo los responsables de Recursos Humanos. Para ellos supone una medida facilitadora del teletrabajo desde casa, para nosotros representa la preservación de los expedientes en soporte digital. Todos ganamos.

\section{Fernando Martín Rodríguez (Biblioteca universitaria)}

Creo que es pronto para determinar cómo va a afectar a largo plazo a la biblioteca en su actividad presencial la situación en la que nos encontramos. Considero que nos tenemos que esforzar en estos momentos por hacer a la biblioteca visible, insistir a los usuarios en que seguimos estando aquí, que somos un lugar en el que además de encontrar información para su proceso de aprendizaje, somos un excelente lugar donde encontrarnos con otros compañeros de la Universidad, poder socializar y en definitiva actuar como un punto de encuentro capaz de crear cultura universitaria. Considero que para una experiencia universitaria plena y completa (cuando los alumnos de motu proprio han desechado las opciones de formación on-line, completamente válidas) la presencialidad es irrenunciable, y en esto la Biblioteca tiene que poner en valor sus espacios e instalaciones como un escenario esencial de la vida universitaria.

Iremos viendo con el tiempo si hemos ganado esta batalla a la "nueva normalidad", pero, de momento, tenemos que reivindicarnos.

María José Lamas (Biblioteca escolar) 
Dados los protocolos a seguir como consecuencia de la pandemia, la biblioteca escolar ve reducida su capacidad para realizar los servicios que antes prestaba. Se mantendrá la posibilidad de realizar préstamos bibliográficos atendiendo a las necesidades de toda la comunidad educativa, pero su funcionamiento se verá limitado en cuanto a la presencia de usuarios en el espacio físico de la misma. En este sentido, está en el mismo estado que poseía en el período de confinamiento. Solamente el profesorado podrá llevar puntualmente a un número reducido de alumnos/as a la biblioteca para la realización de las tareas escolares que lo requieran; no estará abierta durante los períodos de recreo como era habitual para la realización de actividades y consultas; no se realizarán talleres literarios, conferencias, etc. por limitación de aforo.

No obstante, se mantendrá la información y publicitación del material bibliográfico de nueva adquisición, el servicio de préstamo y la disposición necesaria para que toda la comunidad educativa cuente con este servicio con todo su rendimiento en la medida de lo posible.

\section{Carlos García-Romeral Pérez (Biblioteca pública)}

Los Servicios de Lectura Pública son servicios de proximidad, los ciudadanos van a satisfacer sus necesidades emocionales, sociales, aprendizaje y culturales. Son Centros que establecen redes colaborativas en sus distritos y municipios. La "Nueva Normalidad", si la entendemos como la apertura de los servicios con las restricciones, se marca desde la Consejería de Sanidad de la Comunidad de Madrid. Su mayor repercusión no está en el manejo de las colecciones, ni en la sala de lectura, sino en aquellas acciones culturales que desarrollan los Servicios que reúnen a más de 5/6 usuarios o aforos muy limitados: clubes de lectura, proyecciones de cine (cine-fórum), presentaciones de libros... La acción colaborativa de los Servicios con Asociaciones, ONG, Colegios, participación en los medios de comunicación del distrito..., se mantiene más activa y viva.

Este tiempo se aprovecha para el diseño de proyectos que implique de una forma más activa a los ciudadanos.

José Antonio Sánchez Montero (Biblioteca especializada en economía)

Desde mi punto de vista, el gran beneficiado de esta crisis es la industria de contenidos digitales, en tanto que se ha tomado conciencia, más que nunca, de su necesidad. Una vez recuperada la normalidad, "lo digital" va a tener aún mayor presencia que hasta ahora en las políticas de información de la biblioteca; mientras que el teletrabajo consagrará la expansión del uso de los recursos de información en formato electrónico dentro de las organizaciones, para lo que será necesario agilizar el acceso a estos recursos desde cualquier lugar y a través de cualquier dispositivo (móviles, tabletas, portátiles, etc.). Indudablemente, el formato papel seguirá teniendo su parcela de protagonismo, pero cada vez menor, siendo muchas las organizaciones que de poder hacerlo, están migrando sus colecciones impresas al formato electrónico. Todo ello influirá en una menor necesidad de espacios tanto para depósito como de acceso libre para el usuario final en salas de lectura. En relación a las formas de trabajo será necesario ir perfeccionando constantemente los flujos de trabajo y comunicación internas de los equipos de trabajo, en torno a nuevas herramientas digitales.

\section{7. ¿Ha tenido la oportunidad de formarse: cursos, webinarios...?}

Montaña Vivas Jiménez (Biblioteca especializada en ciencias de la salud) 
En este sentido ha sido también una experiencia muy interesante porque la fórmula de cursos y seminarios webs puestos en marcha han sido numerosos y muy interesantes.

El fenómeno del acceso abierto a la mayoría de las publicaciones y a productos de información de las diferentes editoriales y plataformas, ha facilitado enormemente la formación, pero la más importante ha venido de los propios compañeros que han puesto todos sus conocimientos, herramientas, tutoriales, etc. a disposición de todos de forma sencilla.

José Luis Muñoz Romano (Archivo estatal)

Más bien he formado, me ha tocado preparar cuatro cursos, ahora mismo estoy inmerso en la formación de dos, uno de gestión documental avanzada y otro sobre implantación de una política de gestión documental.

Fernando Martín Rodríguez (Biblioteca universitaria)

Ha sido un momento en el que ha habido una enorme oferta formativa on-line mediante cursos de todos los formatos y a cualquier hora. En mi caso y en el de mis compañeros hemos tenido oportunidad de realizar cursos de actualización precisamente en el manejo de TICs, sobre todo.

María José Lamas (Biblioteca escolar)

No. No se han ofertado cursos de formación en este sentido en nuestra comunidad autónoma.

\section{Carlos García-Romeral Pérez (Biblioteca pública)}

Desde la plataforma de "Madrid Digital" se han seguido impartiendo cursos de formación on-line para todos los empleados de la Comunidad de Madrid. Así mismo, se ha animado a la autoformación bien por plataforma oficial o por otras.

Muchos trabajadores han realizado cursos de Absysnet, Word, Excel, gestión de equipos, etc.

José Antonio Sánchez Montero (Biblioteca especializada en economía)

Sí, desde luego, hemos tenido esta posibilidad; es más, en nuestro caso, la formación en nuevas herramientas relacionadas con plataformas de e-books y otros recursos electrónicos de información ha sido necesaria para prestar un servicio adecuado. Por otro lado, de cara al usuario, hemos tenido la oportunidad de organizar una sesión de formación general de usuarios en fuentes de información especializada en competencia y mercados regulados, de 4 horas de duración, sobre los recursos electrónicos de información de la Biblioteca; así como un par de sesiones de formación ad-hoc, sobre fuentes de información específicas de uso en la CNMC (bases de datos y portales especializados).

\section{8. ¿Qué ha significado este periodo para su vida profesional?}

Montaña Vivas Jiménez (Biblioteca especializada en ciencias de la salud)

Una de las experiencias más enriquecedoras por el trabajo que de manera espontánea y sin precedentes hemos llevado a cabo los más de 90 bibliotecarios a través de la iniciativa \#AyudaBiblioteca. La consecuencia ha sido descubrir a muchos colegas de las bibliotecas de ciencias 
de la salud, constatar la fortaleza de nuestro trabajo cuando es colaborativo, y por último dar visibilidad y relevancia al trabajo que desarrollamos y que en este caso hemos sido capaces de proporcionar en tiempo real.

El esfuerzo de las bibliotecas que permanecían abiertas para apoyar a las que se cerraron y atender las necesidades de cualquier profesional que lo necesitara en cualquier punto del país e incluso del extranjero, ha sido enorme pero muy satisfactorio.

Los bibliotecarios que se quedaron sin espacio físico o no pudieron trabajar en sus centros por culpa de la pandemia, nos organizamos creando la red que mencionamos más arriba y que a través del whatsapp intercambiábamos información y recursos de forma vertiginosa y a cualquier hora del día. La demanda se disparó y para organizar la ingente cantidad de documentos se montó un repositorio COVID-19 por especialidades y temas, que rápidamente se convirtió en referente. Se tradujeron artículos del chino, se contactó con profesionales extranjeros que enviaron documentos e información, se podía responder casi a demanda y en muy poco espacio de tiempo..., en definitiva, nos dimos cuenta de la fuerza, la necesidad y la respuesta que son capaces y deben dar las bibliotecas de ciencias de la salud ante este y los próximos retos y desafíos sanitarios.

José Luis Muñoz Romano (Archivo estatal)

Una evolución de rendimiento al emplear indicadores en la medición de resultados, planificando en todo momento al fijar de base con los superiores y tus empleados los objetivos a seguir. Sin embargo, reconozco que ha sido muy estresante, si unimos la situación para nada "normal" de vivir una pandemia, con familiares pasando y afortunadamente superando la COVID-19, sin poder verlos, conciliando 24 horas con dos niños - de 8 y 4 años- que no podían salir de casa durante buena parte de este periodo, teletrabajar ha sido más bien desesperante.

Fernando Martín Rodríguez (Biblioteca universitaria)

He tenido la oportunidad de trabajar con compañeros tanto de la biblioteca de la UBU como de otros servicios y departamentos o de otras bibliotecas, con el empeño de tratar de seguir manteniendo los servicios universitarios. Compañeros prestando equipos informáticos desde sus casas, yendo a trabajar con grandes restricciones durante los primeros momentos de la desescalada para suministrar información a los investigadores, llamadas de teléfono, mensajes de whatsapp y correos atendidos a cualquier hora del día y un enorme esfuerzo por parte de todos por adaptar los espacios a las restricciones de aforo, circulación y señalización. He sentido de una forma contundente la vocación y el compromiso de mis compañeros por el servicio público. Esta forma de afrontar el trabajo por parte de todos me ha hecho sentirme enormemente orgulloso de mi profesión.

María José Lamas (Biblioteca escolar)

Incertidumbre, improvisación y falta de apoyo en todos los sentidos.

Carlos García-Romeral Pérez (Biblioteca pública)

Un replanteamiento de la gestión de los servicios. Valorar la comunicación como uno de los factores más relevantes del éxito de las bibliotecas durante la pandemia. No sólo estar presentes sino ser presentes.

José Antonio Sánchez Montero (Biblioteca especializada en economía) 
Sin duda, ha sido una etapa plena de aprendizaje "digital" a la vez que un reto constante ante las adversidades surgidas.

\section{9. ¿Le gustaría añadir algo? ¿Puede contarnos alguna anécdota o vivencia de esta peculiar situación?}

José Luis Muñoz Romano (Archivo estatal)

Sí, me causó especial impresión el primer día que acudí al Archivo en presencial, prácticamente sin personal en un edificio de la magnitud de Nuevos Ministerios, la soledad, las caras de preocupación de la gente de seguridad y mantenimiento, encontrarte todo cerrado, sin nadie en tu planta... Espero no repetir esa escena.

\section{Fernando Martín Rodríguez (Biblioteca universitaria)}

Durante el estado de alarma, después de las vacaciones de Semana Santa, tuve que acercarme a la Biblioteca a por equipos informáticos para el préstamo, y aprovechando el viaje, tomé prestado un libro que necesitaba una profesora. Quedamos en una de las franjas horarias en la que los dos podíamos coincidir para en un punto intermedio de no más de un kilómetro pasarle el libro sobre derecho financiero. Casi de forma clandestina. Muy poético diría yo...

\section{María José Lamas (Biblioteca escolar)}

Las bibliotecas escolares, a pesar de su importancia a todos los niveles en cuanto al hecho educativo, siguen siendo abandonadas y menospreciadas desde las administraciones educativas ignorando su capacidad para conseguir muchos de los objetivos educacionales. Su función como centro de documentación es la única apreciada, ignorando la faceta de socialización, la de compensación de desigualdades sociales, la de centro de ocio y disfrute por parte del alumnado -más que comprobado en los casi quince años que llevo a cargo de la misma- $y$, sobre todo, como motor dinamizador para consolidar nuevos lectores/as.

Como anécdota, contar que durante muchos años la biblioteca escolar del colegio se convirtió en un lugar deseado por el alumnado en donde se sentía sujeto de la educación y en el cual podía socializar, informarse, formarse y contar con el apoyo necesario para complementar su aprendizaje, de todo lo que estoy orgullosa como coordinadora de este servicio esencial.

\section{Carlos García-Romeral Pérez (Biblioteca pública)}

Lo que más me llama la atención es la responsabilidad y la comprensión de la mayoría de los usuarios ante esta situación. Se echa de menos a los usuarios, no podemos quedar reducidos a un mapa de bit. La sociedad tecnología ha crecido muy rápidamente en poco tiempo, se han organizado talleres filosóficos, de lectura y crecimiento personal utilizando medios y canales por ahora gratuitos. Pero esta tecnología de la "Telepantalla" no humaniza, ni palía la soledad en la que nos estamos encontrando.

$\mathrm{Al}$ abrir las bibliotecas el 3 de junio, en la BP de Usera, un usuario nos dijo, "se os echaba de menos" y le contestamos "y nosotros también a ti".

José Antonio Sánchez Montero (Biblioteca especializada en economía) 
Durante la fase más dura del confinamiento, por primera vez en treinta años de andadura profesional, me he visto obligado a dar una respuesta negativa a un usuario ante su necesidad de acceder al texto completo de un documento. Peor aún, sabiendo que ese mismo documento se hallaba físicamente entre los fondos del depósito de nuestra biblioteca, pero no podía hacer nada para hacérselo llegar.

\section{Conclusiones}

Aunque los archivos y bibliotecas se vieron sorprendidos por la situación del estado de alarma y la pandemia, todos ellos han continuado trabajando y atendiendo a sus usuarios, bien mediante el teletrabajo o presencialmente cumpliendo la normativa. En la desescalada se han seguido o se están siguiendo las normativas generales estatales, autonómicas y las de las propias instituciones; también se han tomado en cuenta las recomendaciones de las entidades profesionales como REBIUN.

En la mayoría de los centros se cerraron o se restringió el uso de las salas de lectura aunque, en general, se ha seguido prestando los servicios básicos, si bien trasladándolos al espacio virtual. Se ha dado una gran importancia al acceso y diseminación de la información y a la potenciación de espacios virtuales de difusión de la cultura o de repositorios colaborativos como el dedicado a la Covid-19 por las bibliotecas de Ciencias de la Salud. Se han reforzado las colecciones digitales, en especial de libros electrónicos, cuyo uso no estaba tan implantado como las revistas electrónicas. En algún caso se ha llegado a implementar un sistema de préstamo y entrega a domicilio.

La pandemia ha supuesto el desarrollo o la adaptación de nuevas formas de trabajo y comunicación, en especial en los centros que tenían mayoritariamente servicios presenciales y fondos en papel. Además, los profesionales han mostrado su gran flexibilidad colaborando en caso necesario con otras áreas de la misma institución.

Los canales de comunicación que se han utilizado han sido muy variados, desde las páginas web, los formularios de consulta o el correo electrónico, hasta herramientas de las redes sociales: cuentas de Twitter, de WhatsApp, blogs, chats. En el caso de las bibliotecas que sirven a los usuarios internos, de la misma institución, se han utilizado herramientas como los sistemas de videollamada o de compartición de ficheros de las intranets. Y en los archivos se ha utilizado la cita previa para mejorar las prestaciones a los usuarios externos. Además, el teletrabajo ha modificado los procesos de trabajo y la comunicación interna entre los equipos.

La pandemia ha facilitado la formación de los profesionales que han seguido cursos de actualización de herramientas concretas relacionadas con el trabajo diario, con fuentes de información, etc. Y a su vez, han tenido que organizar sesiones de formación de recursos de información para los usuarios.

Los profesionales creen que una de las posibles consecuencias del estado de alarma puede ser la reducción de los espacios físicos ya que el mayor desarrollo de fondos y servicios digitales los hará menos necesarios. En el caso de las bibliotecas públicas y escolares, que tienen una mayor orientación a las actividades que implican socialización (cursos, talleres, actividades culturales), impactan especialmente las limitaciones o la no posibilidad de su uso, muy especialmente la biblioteca escolar que, a pesar de su importante papel en la educación, ha sido una parte abandonada de los centros escolares.

En general, nuestros profesionales opinan que la pandemia ha supuesto un reto, un gran esfuerzo de adaptación y colaboración realizado en condiciones muy difíciles en las que se han sentido desbordados y, en algún caso, faltos de apoyo. Pero todos ellos se sienten orgullosos de la respuesta profesional dada a pesar de las dificultades y reflexionan acerca de la necesidad de continuar siendo visibles, relevantes y no renunciar a la presencialidad. 


\section{Entrevistados:}

MONTAÑA VIVAS JIMÉNEZ

Jefe de Sección de la Biblioteca del Complejo Hospitalario Universitario San Pedro de Alcántara. Cáceres

- Directorio Exit: https://www.directorioexit.info/ficha412

- Blog: https://mariamontanavivas.wordpress.com/

JOSÉ LUIS MUÑOZ ROMANO

Jefe de Servicio del Archivo del Ministerio de Fomento

- LinkedIn: https://es.linkedin.com/in/jose-luis-mu\%C3\%B1oz-romano-5787b824

FERNANDO MARTÍN RODRÍGUEZ

Director de la Biblioteca de la Universidad de Burgos

- Perfil: https://investigacion.ubu.es/investigadores/37661/detalle

MARÍA XOSÉ LAMAS

Profesora de Primaria y Encargada de la Biblioteca Escolar Mato Vizoso de Vilalba (Lugo)

- Wikipedia: https://gl.wikipedia.org/wiki/Mar\%CC3\%ADa Xos\%C3\%A9 Lamas

\section{CARLOS GARCÍA-ROMERAL PÉREZ}

Jefe del Área de Servicios Públicos de Lectura de la Comunidad de Madrid

Doctor en literatura. Facultativo de Bibliotecas de la Comunidad de Madrid. Jefe de Área de los Servicios Públicos de Lectura de la Comunidad de Madrid. Bibliográfo

- Correo: carlos.garciaromeral@madrid.org

JOSÉ ANTONIO SÁNCHEZ MONTERO

Responsable del Área de Recursos Documentales. Subdirección de Estadísticas y Recursos Documentales. Comisión Nacional de los Mercados y la Competencia (CNMC)

- Directorio Exit: https://www.directorioexit.info/ficha2978

- LinkedIn: https://www.linkedin.com/in/joseantoniosanchezmontero 